\title{
Antimicrobial stewardship and bamlanivimab: Opportunities for outpatient preauthorization?
}

\author{
Payal K. Patel MD, MPH ${ }^{1}$ (1), Priya Nori MD² and Michael P. Stevens MD, MPH ${ }^{3}$ \\ ${ }^{1}$ Infectious Diseases Section, Ann Arbor Veterans' Affairs Medical Center, Ann Arbor, Michigan, ${ }^{2}$ Division of Infectious Diseases, Department of Medicine, \\ Montefiore Medical Center, Albert Einstein College of Medicine, Bronx, New York and ${ }^{3}$ Healthcare Infection Prevention Department, Virginia Commonwealth \\ University Health System, North Hospital, Richmond, Virginia
}

To the Editor-Preauthorization is a fundamental action of antimicrobial stewardship programs (ASPs). ${ }^{1}$ ASPs have played essential roles in coronavirus disease 2019 (COVID-19) response efforts since the onset of the pandemic. For instance, ASPs have implemented the preauthorization of remdesivir throughout its path from an experimental antiviral obtained via compassionate use or expanded access, to Food and Drug Administration (FDA) Emergency Use Authorization (EUA), to ultimate FDA approval., ${ }^{2,3}$ On November 9, 2020, the FDA released an EUA for bamalanivimab, a recombinant human monoclonal antibody to the spike protein of SARS-CoV-2. ${ }^{4}$ Unlike previous EUAs for remdesivir and convalescent plasma, the bamalanivimab EUA is intended for use in outpatients with mild-to-moderate COVID-19 to avoid hospitalizations due to disease progression. ${ }^{4}$ What role, if any, should ASPs play in bamlanivimab preauthorization? Notably, preauthorization is not commonly deployed by ASPs in outpatient stewardship efforts ${ }^{5}$.

ASPs are well suited to lead preauthorization efforts given their existing experience and infrastructure. ${ }^{2}$ Preauthorization is crucial for a scarce experimental product with limited available supporting evidence in high public demand. Preauthorization is also a critical component of equitable distribution to patients who may maximally benefit. For this process to function optimally, a 24-7 preauthorization structure is ideal but likely is not feasible at most centers. Importantly, time invested in outpatient bamalanivimab preauthorization will siphon effort away from other essential ASP functions. ${ }^{3}$

Another critical role ASPs can play is to lead discussions about the ideal deployment of bamalanivimab locally. Given the limited available literature, including several inpatient studies of monoclonal antibodies that were halted due to unfavorable data, ${ }^{6-8}$ it may be prudent to await further data and/or guidance from professional organizations (eg, the Infectious Diseases Society of America). See Figure 1 for additional considerations.

The bamlanivimab EUA may present ASPs with a golden opportunity to enhance their outpatient stewardship impact. As of January 1, 2020, the Joint Commission has mandated that health systems deploy active antimicrobial stewardship interventions in ambulatory settings. ${ }^{9}$ Bamlanivimab preauthorization can serve as a vehicle for programs to meet the new requirements if administered in outpatient clinics. Beyond this, the infrastructure developed may be harnessed later for other high-value outpatient stewardship targets (eg, long-acting anti-MRSA agents and oral carbapenems). ASPs can develop or strengthen collaborations with emergency departments or urgent-care centers to create a SARSCoV-2 test, treat, and release pathway. In turn, this could help relieve inpatient overcrowding, which has been a national issue in the pandemic. Additionally, telehealth platforms can be utilized for close patient follow-up, potentially increasing revenue and saving costs for hospitals and ASPs.

Perhaps the most critical role of antimicrobial stewards at this juncture is to instill experience and expertise into the process of evaluating the role of bamlanivimab in patient care. If deployed, ASPs can help identify who, when, and where the drug should be given to, and they can help assure compliance with identified best practices. This EUA is further evidence that health systems should invest in their ASPs, for which roles are expanding with each new agent in the COVID-19 armamentarium. 


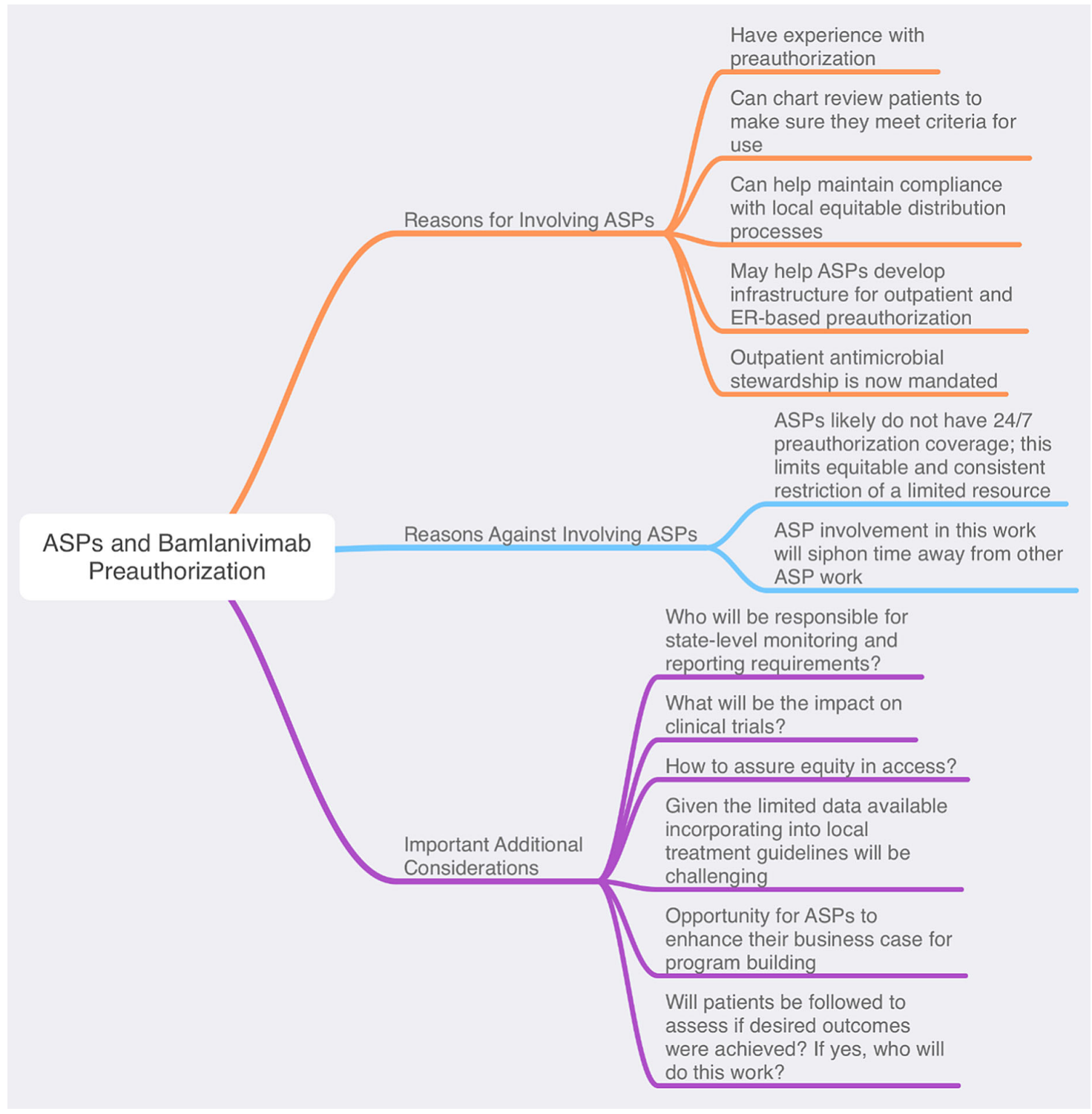

Figure 1. ASPs and Bamlanivimab preauthorization

\section{Acknowledgments.}

Financial support. No financial support was provided relevant to this article.

Conflicts of interest. All authors report no conflicts of interest relevant to this article.

\section{References}

1. Dellit TH, Owens RC, McGowan JE, et al. Infectious Diseases Society of America and the Society for Healthcare Epidemiology of America guidelines for developing an institutional program to enhance antimicrobial stewardship. Clin Infect Dis 2007;44:159-177.

2. Stevens MP, Patel PK, Nori P. Involving antimicrobial stewardship programs in COVID-19 response efforts: all hands on deck. Infect Control Hosp Epidemiol 2020;41:744-745.
3. Mazdeyasna H, Nori P, Patel P, et al. Antimicrobial stewardship at the core of COVID-19 response efforts: implications for sustaining and building programs. Curr Infect Dis Rep 2020;22(9):23.

4. Coronavirus (COVID-19) update: FDA authorizes monoclonal antibody for treatment of COVID-19. US Food and Drug Administration website. https:// www.fda.gov/news-events/press-announcements/coronavirus-covid-19update-fda-authorizes-monoclonal-antibody-treatment-covid-19. Published November 9, 2020. Accessed November 20, 2020.

5. Drekonja DM, Filice GA, Greer N, et al. Antimicrobial stewardship in outpatient settings: a systematic review. Infect Control Hosp Epidemiol 2015;36:142-152.

6. Chen P Nirula A, Heller B, et al. SARS-CoV-2 neutralizing antibody LYCoV555 in outpatients with COVID-19. N Eng J Med 2020. doi: 10.1056/ NEJMoa2029849.

7. REGN-COV2 independent data monitoring committee recommends holding enrollment in hospitalized patients with high oxygen requirements and 
continuing enrollment in patients with low or no oxygen requirements. Regeneron website. https://investor.regeneron.com/news-releases/newsrelease-details/regn-cov2-independent-data-monitoring-committee-

recommends/. Published October 30, 2020. Accessed November 10, 2020.

8. Statement-NIH-sponsored ACTIV-3 trial closes LY-CoV555 substudy. National Institute of Allergy and Infectious Diseases website. https://www. niaid.nih.gov/news-events/statement-nih-sponsored-activ-3-trial-closes-lycov555-sub-study. Published October 26, 2020. Accessed November 10, 2020.

9. R3 Report Issue 23: Antimicrobial stewardship in ambulatory health care. The Joint Commission website. https://www.jointcommission.org/ standards/r3-report/r3-report-issue-23-antimicrobial-stewardship-inambulatory-health-care/. Published 2020. Accessed November 10, 2020.

\title{
Evaluation of an intervention to reduce fomite-mediated transmission of viruses in a simulated restaurant setting
}

\author{
Heba Alhmidi MD ${ }^{1}$, Lucas D. Jones BS², Basya S. Pearlmutter BS ${ }^{1}$, Jennifer L. Cadnum BS ${ }^{1}$, Sandra Y. Silva MD ${ }^{3}$ and \\ Curtis J. Donskey MD ${ }^{4,5}$ \\ ${ }^{1}$ Research Service, Louis Stokes Cleveland Veterans' Affairs (VA) Medical Center, Cleveland, Ohio, ${ }^{2}$ Department of Molecular Biology and Microbiology, Case \\ Western Reserve University School of Medicine, Cleveland, Ohio, ${ }^{3}$ Clinical and Translational Science Program, School of Medicine, Case Western Reserve \\ University, Cleveland, Ohio, ${ }^{4}$ Geriatric Research, Education, and Clinical Center, Louis Stokes Cleveland VA Medical Center, Cleveland, Ohio and ${ }^{5}$ Case Western \\ Reserve University School of Medicine, Cleveland, Ohio
}

To the Editor-The Centers for Disease Control and Prevention (CDC) recently found that adults with positive severe acute respiratory syndrome coronavirus 2 (SARS-CoV-2) test results were twice as likely to report dining at a restaurant than those with negative SARSCoV-2 test results. ${ }^{1}$ Sharing of meals in staff break rooms has also been reported as a source of exposure in healthcare personnel with coronavirus disease 2019 (COVID-19). ${ }^{2}$ Dining in close proximity to others may be a high-risk activity because masks are removed while eating. ${ }^{1-4}$ Air-conditioned ventilation in restaurants might also facilitate transmission of small aerosolized droplets to distances $>1 \mathrm{~m} .{ }^{5}$

In addition to respiratory droplets, transmission of respiratory viruses may occur due to contact with contaminated surfaces and fomites. ${ }^{6-8}$ To reduce the risk of fomite-mediated transmission, the CDC recommends that restaurants and bars clean shared objects between each use and avoid sharing items such as menus and condiments. ${ }^{3}$ The effectiveness of these measures in preventing transmission of pathogens is unclear. Here, we evaluated an intervention to reduce person-to-person transmission from contaminated fomites in restaurants.

The study protocol was approved by the Institutional Review Board of the Louis Stokes Cleveland VA Medical Center. We conducted a quasi-experimental study of transmission by fomites contaminated with bacteriophage MS2 in a simulated restaurant setting. Bacteriophage MS2 was propagated in Escherichia coli. ${ }^{9}$ The simulated restaurant consisted of a $\sim 8 \mathrm{~m} \times 8 \mathrm{~m}$ room with tables positioned $2 \mathrm{~m}$ apart. The study was divided into control simulations and intervention simulations. Research and healthcare personnel participated as customers and wait staff in the simulated restaurant.

Prior to each simulation, an index customer's hands were contaminated with the benign bacteriophage MS2 by applying a $0.5 \mathrm{~mL}$ solution containing the virus. Simulations were conducted with a higher inoculum ( $10^{6}$ plaque-forming units [PFU])

Author for correspondence: Curtis J. Donskey, E-mail: Curtis. Donskey@va.gov Cite this article: Alhmidi $\mathrm{H}$, et al. (2021). Evaluation of an intervention to reduce fomite-mediated transmission of viruses in a simulated restaurant setting. Infection Control \& Hospital Epidemiology, 42: 1526-1528, https://doi.org/10.1017/ice.2020.1327 intended to mimic a worst-case scenario and a lower inoculum $\left(10^{3} \mathrm{PFU}\right)$ intended to be more typical of real-world contamination levels. Three simulations ( 9 customers) were conducted with the higher inoculum and 4 (12 customers) with the lower inoculum.

For each simulation, wait staff invited the index customer to take a seat and provided a laminated menu. After the menu was reviewed, the wait staff took it to a central counter and returned with a bill. After taking a credit card, the wait staff returned with the final bill and a pen that was used to sign the bill. The index customer's hands and the table and chair, menu, pen and wait staff's hands were sampled using premoistened CultureSwabs (Becton Dickinson, San Jose, CA). The swabs were processed as previously described for quantitative culture of virus particles. ${ }^{9}$

A second customer with no bacteriophage MS2 applied was seated at a second table and the same procedures were followed. The menu and pen were not cleaned between customers. Finally, a third customer was seated at the third table and the same procedures were followed. Wait staff did not perform hand hygiene between customers.

Three simulations ( 9 customers) with an intervention were conducted using the higher bacteriophage MS2 inoculum. The protocol was identical to the protocol for the initial simulations except disposable paper menus were used, credit cards were inserted into a card reader such that the card was not contacted by the wait staff, the pen was disinfected with a disinfectant wipe after each use, and wait staff used alcohol hand sanitizer between tables. Wait staff did not touch the used menus or non-decontaminated pens. The used pen was picked up using a disinfectant wipe and used menus were placed into a designated receptacle. The index customer's table and chair were cleaned with an improved hydrogen peroxide disinfectant wipe, and cultures were collected as previously described to assess the effectiveness of disinfection.

The primary outcome tested was the $\log _{10} \mathrm{PFU}$ bacteriophage MS2 concentrations for customers 2 and 3 for the higher inoculum of MS2 in the control simulations versus the intervention

(C) The Author(s), 2020. Published by Cambridge University Press on behalf of The Society for Healthcare Epidemiology of America. This work is classified, for copyright purposes, as a work of the U.S. Government and is not subject to copyright protection within the United States. This is an Open Access article, distributed under the terms of the Creative Commons Attribution licence (http://creativecommons.org/licenses/by/4.0/), which permits unrestricted re-use, distribution, and reproduction in any medium, provided the original work is properly cited. 